\title{
Validação de metodologia analítica para quantificação de desoxinivalenol em amostras de trigo e produtos a base de trigo por CLAE-UV
}

Validation of analytical method for quantification of deoxynivalenol in wheat and wheat products by HPLC-UV

\author{
J. F. Santos ${ }^{1}$; S. A. Silva ${ }^{2 *}$; G. Z. de Rossi ${ }^{2}$; A. P. Almeida ${ }^{2}$ \\ ${ }^{1}$ Curso de Especialização em Vigilância Laboratorial em Saúde Pública/Unidade Didática Instituto Adolfo Lutz, \\ CEFOR/SUS/SP, 01246-000, São Paulo-SP, Brasil \\ ${ }^{2}$ Núcleo de Contaminantes Orgânicos, Instituto Adolfo Lutz, 01246-000, São Paulo-SP, Brasil \\ * simone.silva@ial.sp.gov.br \\ (Trabalho avaliado e selecionado pela Comissão do III CTOCTA)
}

\begin{abstract}
O desoxinivalenol (DON), principal micotoxina encontrada em trigo e derivados, tem sido associado a surtos de gastroenterite, distúrbios gastrointestinais e diarreia em humanos e animais. Para controle das micotoxinas em alimentos há a necessidade de métodos confiáveis e precisos. Desta forma, o presente trabalho teve como objetivo validar metodologia analítica para análise de DON em amostras de trigo e produtos comercializados na cidade de São Paulo. O método foi validado de acordo com os parâmetros linearidade, recuperação, precisão, limites de detecção (LD) e quantificação (LQ). A extração do DON foi realizada por homogeneização com água deionizada, seguida de centrifugação, filtração e purificação em coluna de imunoafinidade. A separação e quantificação foram conduzidas por cromatografia líquida com detecção por ultravioleta (CLAE-UV). A curva analítica, obtida pelo método dos mínimos quadrados, foi linear com coeficiente de correlação de 0,9995 . A recuperação média foi de $102 \%$ e o coeficiente de variação de $2,4 \%$ para a concentração de $877 \mu \mathrm{g} / \mathrm{kg}$. Os LD e LQ foram de 60 e $200 \mu \mathrm{g} / \mathrm{kg}$, respectivamente. Os cromatogramas obtidos não apresentaram interferências na região do tempo de retenção do DON $(9,7$ min), sendo o método proposto considerado adequado para a quantificação de desoxinivalenol em trigo e produtos a base de trigo.

Palavras-chave: Triticum spp., micotoxinas, cromatografia líquida.
\end{abstract}

Deoxynivalenol (DON), the main mycotoxin found in wheat and its derivatives, has been associated with outbreaks of gastroenteritis, gastrointestinal disorders, and diarrhea in humans and animals. For the control of mycotoxins in food, methods with accuracy and precision are needed. Thus, the present work aimed to validate an analytical methodology for the analysis of DON in wheat and products sold in São Paulo city. The method was validated according to the parameters linearity, recovery, precision, limits of detection (LOD), and quantification (LOQ). DON extraction was performed by homogenization with deionized water, followed by centrifugation, filtration, and purification in an immunoaffinity column. The separation and quantification were performed by liquid chromatography with ultraviolet detection (HPLC-UV). The analytical curve, obtained using the square method, was linear with a correlation coefficient of 0.9995 . The average recovery was $102 \%$ and the variation coefficient was $2.4 \%$ for the concentration of $877 \mu \mathrm{g} / \mathrm{kg}$. The LOD and LOQ were 60 and $200 \mu \mathrm{g} / \mathrm{kg}$, respectively. The chromatograms obtained showed no interference in the DON retention time region $(9.7 \mathrm{~min})$, being the proposed method considered adequate for the quantification of deoxynivalenol in wheat and wheat products.

Keywords: Triticum spp., mycotoxins, liquid chromatography.

\section{INTRODUÇÃO}

O trigo (Triticum spp.) é um dos produtos fundamentais para a alimentação humana, e está intimamente relacionado com o surgimento da civilização. Existem cerca de 30.000 variedades de trigo e 14 espécies são cultivadas no mundo. No entanto, somente 1.000 variedades tem importância comercial [1]. É um cereal bem versátil podendo ser utilizado para a produção de vários produtos como farinhas, massas, biscoitos, bolos, pão, e também pode ser incorporado em rações. Além disso, os produtos à base de trigo fazem parte da cesta básica dos brasileiros, devido 
à facilidade de obtenção, simplicidade de processo de fabricação, baixo custo, praticidade e fonte de energia.

Segundo dados da Companhia Nacional de Abastecimento (CONAB), a produção média anual do Brasil foi de 5,4 milhões de toneladas nos últimos 10 anos, no entanto, a quantidade produzida não supre a demanda interna. Os principais produtores são os estados do Paraná e Rio Grande do Sul, que atualmente são responsáveis por aproximadamente $90 \%$ da produção brasileira de trigo. De janeiro a dezembro de 2018 o Brasil importou aproximadamente 6,8 milhões de toneladas de trigo e os principais países exportadores foram Argentina (87,13\%), Paraguai (4,99\%), Estados Unidos $(4,01 \%)$, Canadá $(2,89 \%)$, Uruguai $(0,45 \%)$, e outros $(0,53 \%)$ [2].

Embora o trigo e seus produtos representem uma importante fonte de alimento, essa cultura pode ser afetada por diversos micro-organismos, especialmente os fungos toxigênicos, capazes de provocar doenças como a giberela ou fusariose, responsáveis pela redução da qualidade e rendimento das culturas de trigo e produção de micotoxinas [3].

As micotoxinas são metabólitos secundários produzidos por diversos fungos filamentosos, dentre eles destacam-se os gêneros Aspergillus, Penicillium, Fusarium, Alternaria, Stachibotrys e Claviceps. Na década de 60 começou a despertar interesse no meio científico sobre suas implicações para a saúde humana e animal após a morte de 100.000 perus na Inglaterra que consumiram ração contendo amendoim contaminado por aflatoxinas. Estima-se que mais de 1.000 micotoxinas já foram identificadas e esse número possivelmente crescerá devido ao aumento de publicações, e maior interesse dos centros de pesquisa nos últimos anos [4].

As micotoxinas podem ocorrer nas etapas de pré-colheita, colheita, secagem, armazenamento ou transporte [3]. Segundo a Organização das Nações Unidas para Agricultura e Alimentação (FAO) estima-se que anualmente cerca $25 \%$ das plantações estejam contaminadas com micotoxinas. Isso é preocupante, pois a presença desses contaminantes acima de níveis aceitáveis pode resultar em perdas econômicas significativas para os países, tais como: redução do rendimento e valor das culturas, perda da produtividade animal e custos relacionados à saúde humana [5].

A produção de micotoxinas depende de fatores intrínsecos (características próprias dos alimentos) e fatores extrínsecos (condições ambientais) dentre eles, destacam-se: região de cultivo, composição do alimento, temperatura, $\mathrm{pH}$, atividade de água, umidade relativa do ar, teor de umidade dos alimentos e danos mecânicos [6].

As micotoxinas podem afetar mais de um sistema alvo em uma determinada espécie e os animais podem demonstrar diferenças na susceptibilidade, dependendo dos fatores genéticos (espécies, raças e linhagens), fisiológicos (idade, nutrição, susceptibilidade às doenças) e ambientais (condições climáticas e de manejo), inerentes de cada uma [7].

Segundo a Agência Internacional de Pesquisa em Câncer (IARC), cinco micotoxinas foram consideradas como de maior risco à saúde humana e animal: aflatoxinas, ocratoxina $\mathrm{A}$, zearalenona (ZEA), desoxinivalenol (DON) e fumonisinas [8]. Essas micotoxinas têm recebido maior atenção, devido à frequente ocorrência de efeitos adversos à saúde dos homens e dos animais. A exposição aos seus metabólitos tóxicos pode provocar carcinogênese, teratogênese, imunossupressão e quadros clínicos de neurotoxicidade, nefrotoxicidade, hepatotoxicidade, mielotoxicidade, toxicidade pulmonar e endócrina. A ingestão, inalação ou contato dermal com micotoxinas podem provocar efeitos agudos e crônicos na saúde de seres humanos e animais, conhecidos como micotoxicoses [9].

A principal micotoxina encontrada no trigo e produtos à base de trigo é o DON, que é responsável por provocar efeitos tóxicos em seres humanos e animais, embora seja classificada no grupo 3 da IARC, como não carcinogênica para humanos [8]. Também conhecida como "vomitoxina", é produzida por espécies de Fusarium, resiste a altas temperaturas e pode se manter estável mesmo após o cozimento. Contudo, a concentração desse contaminante pode reduzir consideravelmente em alimentos cozidos na água, como é o caso do macarrão, pois DON é solúvel no meio aquoso [10].

A ingestão é a via mais comum de exposição a esse contaminante. Os efeitos tóxicos associados ao DON são mais evidentes em animais, principalmente em suínos. O DON pode induzir em animais: vômito; causar diarreia e efeitos imunossupressivos; interferir na síntese de proteínas; provocar hemorragia; anorexia, diminuir o ganho de peso e afetar o crescimento. Os 
principais efeitos agudos observados em seres humanos foram: diarreia, náuseas, vômito, dor abdominal, dor de cabeça, tontura e febre [8].

Diante desse cenário é preciso um controle maior das micotoxinas em produtos agrícolas com o intuito de minimizar os riscos em seres humanos e animais. Assim, o ideal é que os contaminantes estejam ausentes ou em quantidades aceitáveis, seguras e de acordo com a legislação vigente. A Agência Nacional de Vigilância Sanitária (ANVISA) publica suas normas com base em dados científicos, considerando as demandas internas e o cenário internacional. No entanto, o setor produtivo precisa de um período para a melhoria de seus processos, sem onerar demasiadamente o consumidor e diminuir a competitividade no mercado. Logo, tanto os órgãos governamentais quanto os produtores precisam ter bom senso na tomada de decisão, levando-se em conta, principalmente, a saúde do consumidor.

A ANVISA, em 18 de fevereiro de 2011, publicou a Resolução da Diretoria Colegiada (RDC) $\mathrm{n}^{\circ} 7$, dispondo sobre limites máximos tolerados (LMT) para micotoxinas em alimentos prontos para oferta ao consumidor e em matérias-primas. Nessa Resolução foram especificados, em anexos, prazos escalonados para a aplicação dos LMT em determinados alimentos, assim o setor produtivo poderia se adequar. Entretanto, em 2013 a ANVISA publicou a RDC n ${ }^{\circ} 59$, de 26 de dezembro de 2013, que prorrogou até 1 de janeiro de 2017 os prazos da RDC no 7 . Em 2017, a RDC $\mathrm{n}^{\circ} 138$, de 8 de fevereiro de 2017 alterou a RDC $\mathrm{n}^{\circ} 7$ estabelecendo mudanças nos LMT de micotoxinas em alimentos como o trigo e produtos de trigo prontos para oferta ao consumidor. Recentemente, a Instrução Normativa IN $n^{\circ}$ 88, de 26 de março de 2021, aplicada de maneira complementar a Resolução RDC $n^{\circ} 487$, de 26 de março de 2021, estabelece os LMT de contaminantes em alimentos, e os valores para DON em produtos de trigo permaneceram os mesmos do início da aplicação da RDC $n^{\circ} 138 / 2017$, com exceção para trigo, milho e cevada, no qual o limite foi reduzido de 3000 para $2000 \mu \mathrm{g} / \mathrm{kg}$, como consta no Quadro 1 [11-15].

Quadro 1: Limites Máximos Tolerados (LMT) estabelecidos para o desoxinivalenol (DON) em alimentos segundo ANVISA.

\begin{tabular}{|cccc|}
\hline & \multicolumn{3}{c|}{ Limites Máximos Tolerados ( $\mathbf{\mu g} / \mathbf{k g})$} \\
\cline { 2 - 4 } & $\begin{array}{c}\text { Aplicação em } \\
01 / 01 / 2017^{\mathrm{a}}\end{array}$ & $\begin{array}{c}\text { Aplicação em } \\
01 / 01 / 2019^{\mathrm{a}}\end{array}$ & $\begin{array}{c}\text { Aplicação em } \\
03 / 05 / 2021^{\mathrm{b}}\end{array}$ \\
\hline $\begin{array}{c}\text { Trigo e milho em grãos para } \\
\text { posterior processamento }\end{array}$ & 3000 & 3000 & 2000 \\
$\begin{array}{c}\text { Trigo integral, trigo para quibe, } \\
\text { farinha de trigo integral, farelo de } \\
\text { trigo, farelo de arroz e grão de } \\
\text { cevada }\end{array}$ & 1250 & 1000 & 1250 \\
$\begin{array}{c}\text { Farinha de trigo, massas, crackers, } \\
\text { biscoitos, produtos de panificação, } \\
\text { cereais e produtos de cereais exceto } \\
\text { trigo }\end{array}$ & 1000 & & \\
\hline
\end{tabular}

${ }^{\mathrm{a}} \mathrm{RDC} \mathrm{n}^{\mathrm{o}} 138 / 2017 ;{ }^{\mathrm{b}} \mathrm{IN} \mathrm{n}{ }^{\mathrm{o}} 88 / 2021$ e RDC n ${ }^{\circ} 487 / 2021$

Desta forma, para que sejam avaliados os limites estabelecidos pela legislação, é necessário o desenvolvimento de métodos de análise seguros que garantam a qualidade analítica dos resultados. Os métodos instrumentais para detectar e quantificar o DON em trigo e cereais incluem a cromatografia líquida de alta eficiência (CLAE) e cromatografia líquida de ultra eficiência (UPLC), com detecção por ultravioleta (UV), cromatografia gasosa, cromatografia líquida acoplada a espectrômetro de massas, ensaio imunoenzimático (ELISA) e espectroscopia de infravermelho próximo [16]. A disponibilização de metodologias de análise precisas poderá determinar o grau de contaminação dos alimentos por micotoxinas, bem como a avaliação da exposição da população ao risco e a confirmação do diagnóstico de micotoxicoses $[17,18]$. 
Este trabalho teve como objetivo o desenvolvimento e a validação de metodologia analítica para a determinação de desoxinivalenol em amostras de trigo e produtos a base de trigo (trigo em grão, farelo de trigo, trigo para quibe e farinha de trigo) por cromatografia líquida de alta eficiência com detecção por ultravioleta (CLAE-UV).

\section{MATERIAL E MÉTODOS}

\subsection{Materiais e reagentes}

Os reagentes utilizados foram metanol (Sigma Aldrich; St. Louis, MO, EUA) e acetonitrila (J.T. Baker; Avantor Inc.; Radnor, PA, EUA), ambos grau CLAE. O padrão DON foi adquirido da Sigma Chemicals Company (St. Louis, MO, EUA), a coluna de imunoafinidade (CIA) DonStar ${ }^{\mathrm{TM}}$ da empresa Romer Labs (Union, MO, EUA), o material de referência certificado de trigo triturado B-MYC0856 da empresa LGC Standards (Wesel, Alemanha) com valor certificado de $877 \pm 23 \mu \mathrm{g} / \mathrm{kg}$, e a água utilizada foi ultra-purificada por um sistema Synergy UV (Millipore SAS, Molsheim, França).

O padrão DON foi dissolvido em acetonitrila e armazenado a $-18{ }^{\circ} \mathrm{C}$ em um frasco selado em escuro até o uso. A concentração foi determinada por um espectrofotômetro (8453, Hewlett Packard; Santa Clara, CA, EUA) a $217 \mathrm{~nm}$ segundo procedimento descrito em Instituto Adolfo Lutz (2008) [19]. A solução padrão foi utilizada para preparar soluções de trabalho em água ultrapura para construção da curva analítica de DON em sete diferentes níveis de concentração: $50,100,200,400,600,800$ e $1000 \mathrm{ng} / \mathrm{mL}$. A quantificação foi realizada por padronização externa. Amostras de trigo com baixa concentração de DON foram utilizadas para a validação do método cromatográfico.

\subsection{Extração e purificação}

A metodologia foi fundamentada no estudo de Almeida et al. (2016) [20]. As amostras foram previamente trituradas em liquidificador industrial (Vitaprep, Vitamix Corporation; Ohio, EUA). Cada amostra foi identificada e armazenada em temperatura ambiente. As amostras foram homogeneizadas e pesou-se uma porção de $12,5 \mathrm{~g}$ da amostra que foi extraída com $100 \mathrm{~mL}$ de água ultrapura em agitador orbital (KS 501 Digital; IKA Labortechnik; Staufen, Alemanha) por $30 \mathrm{~min}$ a $300 \mathrm{rpm}$. Após a agitação aguardou-se a decantação da amostra, o sobrenadante foi transferido para um tubo de $50 \mathrm{~mL}$ e centrifugado a $3000 \mathrm{rpm}$ por $10 \mathrm{~min}$ (Nova Técnica; Centrífuga NT 812, Piracicaba, Brasil). O extrato foi filtrado através de papel de microfibra de vidro $(0,45 \mu \mathrm{m})$ e coletado em um tubo de ensaio

A etapa de limpeza foi realizada no sistema automatizado de extração em fase sólida (GX-274 ASPEC, Gilson, Inc.; Middleton, WI, EUA). O carregamento do extrato para a CIA DonStar ${ }^{\mathrm{TM}}$ foi de $2 \mathrm{~mL}$, com um fluxo de 1 - 2 gotas/s. Em seguida, a coluna foi lavada duas vezes com 10 $\mathrm{mL}$ de água ultrapura, com fluxo de $2 \mathrm{~mL} / \mathrm{min}$. O excesso de água foi retirado usando concentrador (5301, Eppendorf; Hamburg, Alemanha) a uma velocidade de $1400 \mathrm{rpm}$ por $5 \mathrm{~min}$. Em seguida, a eluição foi realizada no sistema GX-274 ASPEC com $2 \mathrm{~mL}$ de metanol com fluxo de $0,5 \mathrm{~mL} / \mathrm{min}$, o eluato coletado em tubo, e evaporado em banho com temperatura de $45^{\circ} \mathrm{C}$ sob fluxo de $\mathrm{N}_{2}$. O resíduo seco foi dissolvido em $1 \mathrm{~mL}$ de água ultrapura, a solução foi homogeneizada e transferida para vial para posterior análise cromatográfica.

\subsection{Análise por CLAE-UV}

A etapa cromatográfica foi realizada em um Cromatógrafo Líquido de Alta Eficiência (Waters Alliance 2695, Waters Corporation; Milford, MA, EUA) equipado com um detector de UVvisível com comprimento de onda a $220 \mathrm{~nm}$, coluna cromatográfica C18 Microsorb-MV 100-5, 250 x 4,6 mm, $5 \mu \mathrm{m}$ (Varian Medical Systems; Walnut Creek, CA, EUA) e coluna de guarda C18 ZORBAX, 4 × 3 mm (Agilent Technologies; Palo Alto, CA, EUA). A fase móvel utilizada foi água ultra-purificada: metanol: acetonitrila $(80: 15: 5, \mathrm{v} / \mathrm{v} / \mathrm{v})$ a uma taxa de fluxo de $0,8 \mathrm{~mL} / \mathrm{min}$. $\mathrm{O}$ volume de injeção foi de $50 \mu \mathrm{L}$ e o tempo de corrida de 12 minutos. 


\subsection{Desempenho analítico}

A validação do método foi realizada de acordo com os parâmetros de linearidade, recuperação, precisão, limites de detecção (LD) e quantificação (LQ), com base no protocolo descrito pelo Instituto Nacional de Metrologia, Qualidade e Tecnologia [21] e de acordo com os trabalhos de Almeida et al. (2016) [20] e Silva et al. (2020) [22].

A linearidade foi expressa pelo coeficiente de correlação (r) da curva de calibração obtida de sete diferentes níveis de concentração, analisadas em triplicata, de soluções de calibração de padrão externo com concentrações de DON variando de 50 a $1000 \mathrm{ng} / \mathrm{mL}$, equivalente a 200$4000 \mu \mathrm{g} / \mathrm{kg}$ na amostra (fator de diluição x4).

As médias de recuperação foram calculadas com base em seis replicatas do material de referência certificado (MYC0856) em um único nível de concentração $(877 \pm 23 \mu \mathrm{g} / \mathrm{kg}$ ). A precisão (repetibilidade e precisão intermediária) foi avaliada usando o coeficiente de variação (CV) durante os testes de recuperação no mesmo dia e em dias alternados, realizados por dois analistas diferentes.

Os valores de LD e LQ foram estimados na razão do sinal/ruído ( $\mathrm{S} / \mathrm{N})$ de 3 e 10 vezes, respectivamente. O limite de quantificação corresponde ao primeiro ponto da curva de calibração.

A precisão também foi avaliada por participação em teste de proficiência organizado pela BRPAS (Brasil Amostras Analíticas de Proficiência - BR0001/2010) para medição DON em farinha de trigo.

\section{RESULTADOS E DISCUSSÃO}

\subsection{Concentração da solução padrão}

A absorbância foi medida em triplicata no comprimento de onda de $217 \mathrm{~nm}$ e a concentração da solução padrão foi obtida utilizando a fórmula da lei de Lambert-Beer [19]:

$$
\text { Concentração }=\frac{\text { Abs média } \times \mathrm{CF} \times \mathrm{MM} \times 1000}{\varepsilon \times \mathrm{b}}
$$

sendo:

Abs média $=$ absorbância média a $217 \mathrm{~nm}(0,9604)$

$\mathrm{CF}=$ fator de correção do espectrofotômetro (1)

$\mathrm{MM}=$ massa molar $(296,32 \mathrm{~g} / \mathrm{mol})$

$\varepsilon=$ absortividade molar do desoxinivalenol em acetonitrila $(6825 \mathrm{~L} / \mathrm{mol} . \mathrm{cm})$

$\mathrm{b}=$ caminho óptico $(1 \mathrm{~cm})$

A concentração real obtida para o DON em solução foi de 41,697 $\mu \mathrm{g} / \mathrm{mL}$. A Figura 1 apresenta o espectro UV do desoxinivalenol.

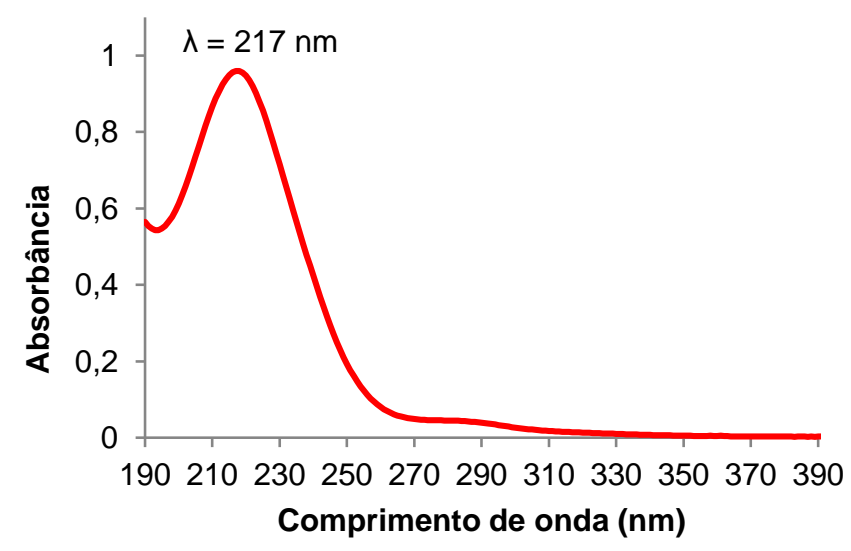

Figura 1: Espectro UV da solução padrão de desoxinivalenol em acetonitrila. 


\subsection{Parâmetros de validação da metodologia}

A curva de calibração de DON, obtida pelo método dos mínimos quadrados, foi linear na faixa de 50-1000 ng/mL. Os dados de validação estão sumarizados na Tabela 2 e a Figura 2 apresenta os cromatogramas dos sete níveis da curva.

Tabela 2: Parâmetros de validação da metodologia de DON para trigo e derivados de trigo.

\begin{tabular}{ccccccc}
\hline $\begin{array}{c}\text { Curva } \\
\text { analítica }\end{array}$ & $\mathbf{r}$ & $\begin{array}{c}\mathbf{L D} \\
(\boldsymbol{\mu g} / \mathbf{k g})\end{array}$ & $\begin{array}{c}\mathbf{L Q} \\
(\boldsymbol{\mu g} / \mathbf{k g})\end{array}$ & $\begin{array}{c}\text { Recuperação }_{\mathbf{M e ́ d i a}^{\mathbf{a}}} \\
(\boldsymbol{\%})\end{array}$ & $\begin{array}{c}\text { CV } \\
\text { Repetibilidade }_{(\boldsymbol{\%})^{\mathbf{a}}}\end{array}$ & $\begin{array}{c}\text { CV Precisão } \\
\text { Intermediária } \\
(\boldsymbol{\%})^{\mathbf{a}}\end{array}$ \\
\hline $\begin{array}{c}\mathrm{y}=70,587 \mathrm{x}- \\
925,141\end{array}$ & 0,9995 & 60 & 200 & 102,0 & 2,4 & 2,8 \\
\hline
\end{tabular}

LD: limite de detecção; LQ: limite de quantificação; $r$ = coeficiente de correlação; ${ }^{a}$ As médias de recuperação e coeficiente de variação $(\mathrm{CV})$ foram calculados com base em seis repetições $(\mathrm{n}=6)$ do material de referência certificado de trigo (B-MYC0856, concentração de DON: $877 \pm 23 \mu \mathrm{g} / \mathrm{kg}$ ).

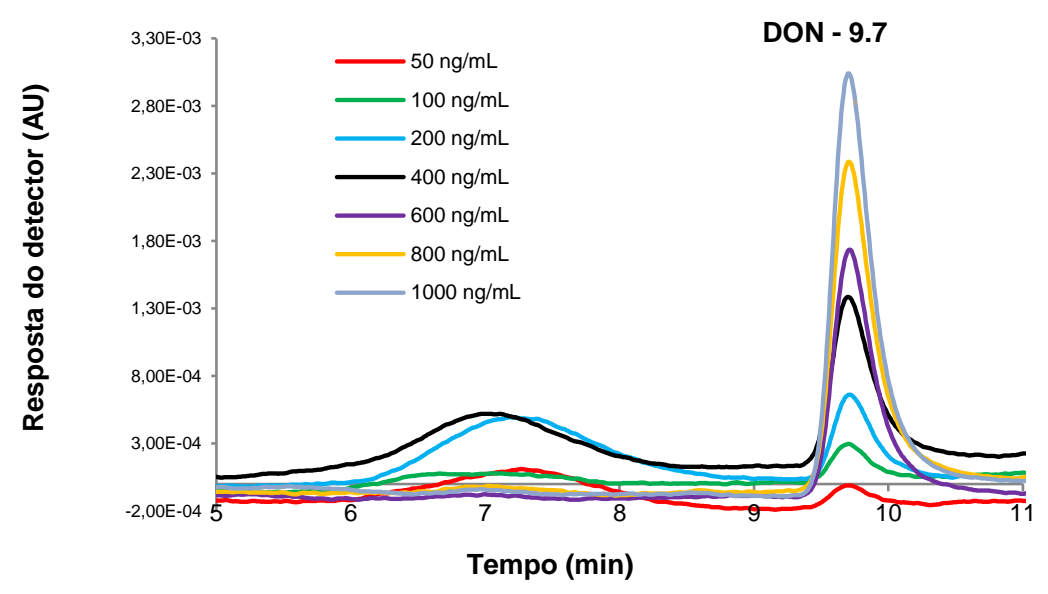

Figura 2: Cromatogramas de DON em diferentes níveis de concentração da curva de calibração.

A exatidão do método foi avaliada através de testes de recuperação, e os resultados variaram de 99,5 a 104,9\%, com uma recuperação média de 102\%, considerado adequado segundo Inmetro (2020) [21]. A recuperação foi superior à faixa encontrada em amostras de trigo avaliadas no Paquistão [23] e na Turquia [24], que variaram de 80,8 a 90,0\% e 85,8 a 90,3\%, respectivamente. $\mathrm{Na}$ avaliação da precisão, realizados no mesmo dia (repetibilidade) e em dias alternados (precisão intermediária), os valores de CV ficaram abaixo de $3 \%$, condizentes com os obtidos por Golge e Kabak (2020) (CV máximo de 5,46\%) [24]. O Z-score obtido no teste de proficiência (BR0001/2010 - DON em farinha de trigo) foi de 0,49, confirmando a precisão da metodologia.

Os valores dos limites de detecção e quantificação foram de 60 e $200 \mu \mathrm{g} / \mathrm{kg}$, respectivamente. O LD encontrado por Iqbal et al. (2020) [23], que usaram o mesmo método (CIA para purificação e CLAE-UV para detecção) foi de $50 \mu \mathrm{g} / \mathrm{kg}$, próximo ao obtido neste estudo, enquanto o LQ foi de $100 \mu \mathrm{g} / \mathrm{kg}$, inferior ao calculado. Os cromatogramas obtidos para a análise dos extratos das amostras não apresentaram pico interferente na região do tempo de retenção do desoxinivalenol $(9,7 \pm 0,2 \mathrm{~min})$ (Figura 3). 


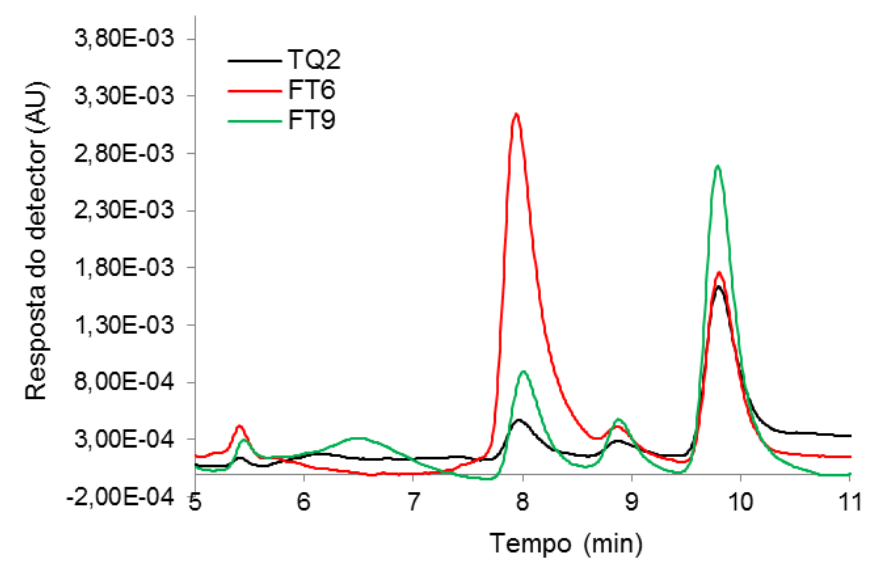

Figura 3: Cromatogramas das amostras de trigo para quibe (TQ2) e farinha de trigo (FT6 e FT9).

\section{CONCLUSÃO}

A determinação de DON por CLAE-UV foi efetuada de modo eficiente, com base na extração em água ultrapura, seguida por uma etapa de purificação e limpeza em coluna de imunoafinidade. Os parâmetros de validação avaliados indicaram que o método é confiável para a análise deste contaminante em amostras de trigo e produtos a base de trigo.

\section{REFERÊNCIAS BIBLIOGRÁFICAS}

1. Posner ES, Hibbs AN. Wheat flour milling. 2. ed. Saint Paul (MN): American Association of Cereal Chemists; 2005.

2. Portal de Informações Agropecuárias [Internet]. [local desconhecido]: CONAB. Jan-Dez 2018 [citado em 06 out 2020]. Disponível em: https://portaldeinformacoes.conab.gov.br

3. Marin S, Ramos A, Cano-Sancho G, Sanchis V. Mycotoxins: Occurrence, toxicology, and exposure assessment. Food Chem Toxicol. 2013;60:218-37. doi: 10.1016/j.fct.2013.07.047

4. Turner NW, Subrahmanyam S, Piletsky SA. Analytical methods for determination of mycotoxins: a review. Anal Chim Acta. 2009;632(2):168-80. doi: 10.1016/j.aca.2008.11.010

5. Matny O. Fusarium head blight and crown rot on wheat \& barley: Losses and health risks. Adv Plants Agric Res. 2015;2(1):00039. doi: 10.15406/apar.2015.02.00039

6. Pitt J, Hocking A. Fungi and food spoilage. 3. ed. New York (US): Springer; 2009.

7. da Rocha MEB, Freire FCO, Maia FEF, Guedes MIF, Rondina D. Mycotoxins and their effects on human and animal health. Food Control. 2014;36(1):159-65. doi: 10.1016/j.foodcont.2013.08.021

8. International Agency for Research on Cancer (IARC), organizadora. Some naturally occurring substances, food items and constituents heterocyclic aromatics amines and mycotoxins. Lyon (FR): IARC; 1993. (IARC Monographs on the evaluation of the carcinogenic risk of chemicals to humans; vol. 56).

9. Gimeno A, Martins ML. Micotoxinas y micotoxicosis en animales y humanos. 3. ed. Florida (US): Special Nutrients, Inc.; 2011. Subitem 4.6, Toxina T-2; p. 50-3.

10. Kushiro M. Effects of milling and cooking processes on the deoxynivalenol content in wheat. Int J Mol Sci. 2008;9(11):2127-45. doi: 10.3390/ijms9112127

11. Brasil. Ministério da Saúde, Agência Nacional de Vigilância Sanitária (ANVISA). Resolução - RDC n ${ }^{\circ}$ 7, de 18 de fevereiro de 2011. Dispõe sobre limites máximos tolerados (LMT) para micotoxinas em alimentos. Diário Oficial da União. 9 mar 2011;46(Seção 1):66-67. Disponível em: https://pesquisa.in.gov.br/imprensa/jsp/visualiza/index.jsp?data=09/03/2011\&jornal=1\&pagina=66\&t otalArquivos $=160$

12. Brasil. Ministério da Saúde, Agência Nacional de Vigilância Sanitária (ANVISA). Resolução - RDC $\mathrm{n}^{\circ}$ 59, de 26 de dezembro de 2013. Dispõe sobre a prorrogação dos prazos estabelecidos nos artigos 11 e 12 e respectivos anexos III e IV da Resolução - RDC n ${ }^{\circ}$, de 18 de fevereiro de 2011 que dispõe sobre os limites máximos tolerados (LMT) para micotoxinas em alimentos. Diário Oficial da União. 30 dez 2013;252(Seção 1):756. Disponível em: https://www.gov.br/agricultura/ptbr/assuntos/inspecao/produtos-vegetal/legislacao-1/biblioteca-de-normas-vinhos-e-bebidas/resolucaordc-no-59-de-26-de-dezembro-de-2013.pdf/view 
13. Brasil. Ministério da Saúde, Agência Nacional de Vigilância Sanitária (ANVISA). Resolução - RDC $n^{\circ}$ 138, de 08 de fevereiro de 2017. Altera a Resolução da Diretoria Colegiada - RDC n ${ }^{\circ}$, de 18 de fevereiro de 2011. Dispõe sobre limites máximos tolerados (LMT) para micotoxinas em alimentos, para alterar os LMT da micotoxina Desoxinivalenol (DON) em trigo e produtos de trigo prontos para oferta ao consumidor e os prazos para sua aplicação. Diário Oficial da União. 9 fev 2017;29(Seção 1):45. Disponível em: https://www.in.gov.br/web/dou/-/resolucao-rdc-n-138-de-8-de-fevereiro-de-201720794496

14. Brasil. Ministério da Saúde, Agência Nacional de Vigilância Sanitária (ANVISA). Instrução Normativa - IN nº 88, de 26 de março de 2021. Estabelece os limites máximos tolerados (LMT) de contaminantes em alimentos. Diário Oficial da União. 31 mar 2021;61(Seção 1):226. Disponível em: https://www.in.gov.br/web/dou/-/instrucao-normativa-in-n-88-de-26-de-marco-de-2021-311655598

15. Brasil. Ministério da Saúde, Agência Nacional de Vigilância Sanitária (ANVISA). Resolução RDC $n^{\circ}$ 487, de 26 de março de 2021. Dispõe sobre os limites máximos tolerados (LMT) de contaminantes em alimentos, os princípios gerais para o seu estabelecimento e os métodos de análise para fins de avaliação de conformidade. Diário Oficial da União. 31 mar 2021;61(Seção 1):225. Disponível em: https://www.in.gov.br/en/web/dou/-/resolucao-rdc-n-487-de-26-de-marco-de-2021-311593455

16. Shen G, Cao Y, Yin X, Dong F, Xu J, Shi J, et al. Rapid and nondestructive quantification of deoxynivalenol in individual wheat kernels using near-infrared hyperspectral imaging and chemometrics. Food Control. 2022;131:108420. doi: 10.1016/j.foodcont.2021.108420

17. Bryden WL. Mycotoxin contamination of the feed supply chain: Implications for animal productivity and feed security. Anim Feed Sci Technol. 2012;173(1-2):134-58. doi: 10.1016/j.anifeedsci.2011.12.014

18. Binder EM. Managing the risk of mycotoxins in modern feed production. Anim Feed Sci Technol. 2007;133(1-2):149-66. doi: 10.1016/j.anifeedsci.2006.08.008

19. Instituto Adolfo Lutz (São Paulo). Métodos físico-químicos para análise de alimentos [Internet]. 4. ed.; 1. ed. digital. São Paulo (SP): Instituto Adolfo Lutz; 2008 [citado em 16 jul 2021]. Disponível em: http://www.ial.sp.gov.br/resources/editorinplace/ial/2016_3_19/analisedealimentosial_2008.pdf

20. Almeida AP, Lamardo LCA, Shundo L, da Silva SA, Navas SA, Alaburda J, et al. Occurrence of deoxynivalenol in wheat flour, instant noodle and biscuits commercialised in Brazil. Food Addit Contam Part B Surveill. 2016;9(4):251-5. doi: 10.1080/19393210.2016.1195880

21. Instituto Nacional de Metrologia, Qualidade e Tecnologia (INMETRO). DOQ-CGCRE-008: Orientação sobre validação de métodos analíticos [Internet]. Revisão 09. Rio de Janeiro: INMETRO; 2020. Disponível em: http://www.inmetro.gov.br/credenciamento/organismos/doc_organismos.asp?tor ganismo $=$ calibensaios

22. Silva SA, Almeida AP, Lamardo LCA, Shundo L, Ruvieri V. GX-274 ASPEC ${ }^{\circledR}$ : Automated Extraction of Deoxynivalenol (DON) from Wheat [Internet]. Middleton (WI): Gilson Inc; 2020 [citado em 06 out 2020]. Disponível em: https://www.gilson.com/pub/media/docs/ASPEC274_ExtractionDeoxynivalen olWheat_AN1012-01.pdf

23. Iqbal SZ, Usman S, Razis AFA, Basheir Ali N, Saif T, Asi MR. Assessment of deoxynivalenol in wheat, corn and its products and estimation of dietary intake. Int $J$ Environ Res Public Health. 2020;17(15):5602. doi: 10.3390/ijerph17155602

24. Golge O, Kabak B. Occurrence of deoxynivalenol and zearalenone in cereals and cereal products from Turkey. Food Control. 2020;110:106982. doi: 10.1016/j.foodcont.2019.106982 